\title{
Comparison of clinical outcomes following 2 years of treatment of first-episode psychosis in urban early intervention services in Canada and India
}

Ashok Malla*, Srividya N. Iyer* , Thara Rangaswamy, Padmavati Ramachandran, Greeshma Mohan, Aarati Taksal, Howard C. Margolese, Norbert Schmitz and Ridha Joober

\section{Background}

Purported superior outcomes for treatment of psychosis in lowand middle-income (LMICs) compared with high-income (HICs) countries have not been examined in the context of early intervention services (EIS).

\section{Aims}

To compare 2-year clinical outcomes in first-episode psychosis (FEP) treated in EIS in Chennai (LMIC) and Montreal (HIC) using a similar EIS treatment protocol and to identify factors associated with any outcome differences.

\section{Method}

Patients with FEP treated in EIS in Chennai $(n=168)$ and Montreal $(n=165)$ were compared on change in level of symptoms and rate and duration of positive and negative symptom remission over a 2-year period. Repeated-measures analysis of variance, and logistic and linear regression analyses were conducted.

\section{Results}

Four patients died in Chennai compared with none in Montreal. Family support was higher for Chennai patients $(F=14.05$, d.f. $=1$, $\left.P<0.001, n p^{2}=0.061\right)$ and increased over time at both sites $\left(F=7.0\right.$, d.f. $\left.=1.915, P<0.001, n p^{2}=0.03\right)$. Negative symptom outcomes were significantly better in Chennai for level of symptoms (time $\times$ site interaction $F=7.36$, d.f. $=1.49, P=0.002$ $\mathrm{np}^{2}=0.03$ ), duration of remission (mean $16.1 \mathrm{~V} .9 .78$ months, $t=$ -7.35 , d.f. $=331, P<0.001$, Cohen's $d=0.80$ ) and the proportion of patients in remission $\left(81.5 \%\right.$ v. $60.3 \%, \chi^{2}=16.12$, d.f. $=1, P<$ $0.001)$. The site differences in outcome remained robust after adjusting for inter-site differences in other characteristics. Early remission and family support facilitated better outcome on negative symptoms. No significant differences were observed in positive symptom outcomes.

\section{Conclusions}

Patients with FEP treated in EIS in LMIC contexts are likely to show better outcome on negative symptoms compared with those in HIC contexts. Early remission and family support may benefit patients across both contexts.

\section{Keywords}

First-episode psychosis; early intervention service; clinical outcomes; high-income low-income country comparison; family support.

\section{Copyright and usage}

(c) The Authors 2020. Published by Cambridge University Press on behalf of the Royal College of Psychiatrists.
Early intervention services (EIS) have been shown to improve outcomes in first-episode psychosis (FEP). ${ }^{1}$ Key components of EIS generally include case management, low-dose second-generation antipsychotics and family intervention. ${ }^{2}$ However, most of the evidence for superior effectiveness of EIS comes from high-income countries (HICs).

Past reports of better outcomes in psychotic disorders in lowand middle-income countries (LMICs) compared with $\mathrm{HICs}^{3-6}$ have been criticised $^{7}$ for not taking into account mortality using standard mortality ratios, the underreporting of suicides in LMICs, the lack of representative samples and inadequately measured outcomes. Despite these criticisms, this observation may hold true for India ${ }^{4}$ for patients who actually receive adequate treatment.

Whether the differences in outcomes reported in the past between India and HICs would persist if similar EIS principles and treatment components were applied in both settings has never been examined. Hypotheses focused on culture ${ }^{8}$ have been proposed to explain any differences in outcome. However, what might facilitate better outcomes within a culture remains unexplored. The impact of overall social support on outcome has been well documented. ${ }^{9}$ A greater role of families, as a specific and deeper form of social support, in India might be one important facilitator, given the context of a family structure in which the

* Joint first authors. individual remains integrated with and supported by the family amidst a general lack of state support. ${ }^{10}$

We have previously confirmed differences in clinical and functional outcomes in a pilot study ${ }^{11}$ using relatively small FEP samples in Montreal (Canada) and Chennai (India). On the basis of these findings, we conducted a larger study across the two sites, comparing multiple outcomes that were established $a$ priori and included clinical, functional and subjective domains. Here we report only on clinical outcomes (symptoms). We hypothesised that (a) following application of key components of EIS, clinical outcomes would be better for patients in Chennai compared with those in Montreal; and (b) that such differences would be explained by differences in previously known predictors of outcomes, especially family support.

\section{Method}

\section{Research and treatment context}

This longitudinal, 2-year prospective outcomes study was conducted between 2012 and 2018 in Montreal (population 3.2 million), Canada, and Chennai (population 6 million), India. Within Montreal's McGill University network, the Prevention and Early Intervention Program for Psychoses (PEPP) is comprised of a larger service situated (since 2003) at the Douglas Mental Health University Institute and a smaller service at the McGill University Health Centre. ${ }^{12}$ The former serves a defined catchment area (total population 300000 ) in south-west Montreal, with a 
significant proportion from minority ethnic groups (30\%), and the latter mostly serves central Montreal (no defined catchment area), with a sizable student population. The two services are attached to a psychiatric and a general hospital respectively, with designated in-patient beds, and operate largely as out-patient community programmes.

Chennai, a large metropolis in South India, has a predominantly Tamil-speaking population with literacy rates of $90 \%$. It hosts the Schizophrenia Research Foundation (SCARF), which is a nongovernmental organisation and a World Health Organization research centre. Not being a catchment area-based service, SCARF accepts patients from all sources and all parts of Chennai. Its capacity for EIS was built collaboratively between 2006 and 2008 and adapted to sensitivities of the Indian cultural context and resource constraints, prior to the pilot project (2008-2010). It does not have an emergency psychiatric service but has access to beds when needed. We acknowledge that neither Chennai nor Montreal can be considered as representing the entire world of urban India or urban Canada respectively.

Both PEPP and SCARF followed EIS protocols for treatment of FEP, i.e. second-generation antipsychotic medications in lowest effective doses, case management, family psychoeducation and individual family intervention, cognitive-behavioural therapy when indicated and an overall recovery orientation. ${ }^{11}$ Services, including medication, were available to patients free of charge at both sites.

All study procedures complied with the ethical standards of the relevant national and institutional committees on human experimentation and with the Helsinki Declaration of 1975, as revised in 2008; and were approved by the Institutional Review Board at SCARF, Chennai, and the Research Ethics Board at McGill University, Montreal.

\section{Participants}

Inclusion criteria: an FEP defined as meeting DSM-IV-TR ${ }^{13}$ criteria for a primary DSM-IV-TR ${ }^{13}$ diagnosis of either schizophreniaspectrum psychotic disorder or affective psychosis irrespective of the time since onset of psychosis, and not having received antipsychotic medication for more than 30 days since the onset of psychosis; age 16-35 years; and ability to communicate fluently in Tamil or English in Chennai, and French or English in Montreal.

Exclusion criteria: IQ below 70; psychosis secondary to a central nervous system disorder (e.g. epilepsy) or a medical condition; and a primary diagnosis of substance dependence. Patients who met the inclusion criteria but had a comorbid diagnosis of substance misuse were included.

Admission criteria for the two EIS were the same as the study entry criteria, except for the Montreal network also admitting 14to 16-year-olds.

Sample size calculation: for clinical outcomes, using pilot data ${ }^{11}$ on symptom assessments, we assumed a difference of $15 \%$ in reduction of positive and negative symptoms between the two samples. We found that a total sample of 200 would allow us to detect a significant time $\times$ site interaction with $90 \%$ power at a type I error of 0.05 . Conservatively factoring in attrition rates of $15 \%$ at SCARF and $24 \%$ at PEPP, we had estimated that a sample of 150 would be required at each site to observe significant differences in outcome. Supplementary Fig. 1, available at https://doi.org/10. 1192/bjp.2020.126, provides details of patient recruitment. All consecutive patients (248 in Montreal; 244 in Chennai) entering the treatment programmes were assessed for inclusion in the study. Those meeting the inclusion criteria were approached for participation in the study. The final sample comprised 333 individuals with previously untreated FEP $(n=168$ in Chennai; $n=165$ in
Montreal). Informed consent was obtained from all participants. Participants were offered a 24-month treatment programme.

\section{Assessments \\ Sociodemographic and clinical information}

Diagnosis was established through the Structured Clinical Interview for DSM-IV (SCID).${ }^{14}$ Sociodemographic information, age at onset of psychosis and duration of untreated psychosis (DUP) were obtained by administering the Circumstances of Onset and Relapse Schedule (CORS). ${ }^{15}$ DUP was defined as the number of weeks between the onset of psychosis and the initiation of antipsychotic medication. The suitability of the CORS in cross-cultural research and interrater reliability between raters at the two sites were established on 10 randomly selected cases (intraclass correlation coefficient ICC $=0.89-0.97$ ) in the pilot study. ${ }^{11}$

\section{Symptom assessments}

The Scale for Assessment of Positive Symptoms (SAPS) ${ }^{16}$ and the Scale for Assessment of Negative Symptoms (SANS) ${ }^{17}$ were used to rate symptoms at baseline and at months $2,3,6,12,18$ and 24 . Positive and negative symptom severity were based on total SAPS and SANS scores respectively. The latter excluded scores on the items of 'inappropriate affect' and 'poverty of content of speech' and the items for the 'attention' subscale, as these have been shown not to be part of the negative symptoms domain. ${ }^{18}$

\section{Medication}

All participants were prescribed a second-generation antipsychotic medication, with the specific choice based on clinical consideration and availability. Prescription of other psychotropic medications was based on clinical need. Adherence to medication was recorded monthly on the basis of information obtained from participants and/or their family members. Participants were regarded as adherent in a given month if they took the antipsychotic medication 76$100 \%$ of the time in that month. This method has been validated and used in other studies. ${ }^{19,20}$ Percentage of adherence was calculated by dividing the number of months that the participant was adherent by the number of months for which they were prescribed antipsychotic medication, multiplied by 100 .

\section{Remission}

The Remission in Schizophrenia Working Group's consensus criteria $^{21}$ were used to define remission. Participants were in positive or negative remission if they scored 2 or less on each global subscale of 'hallucinations, delusions, bizarre behaviour and formal thought disorder' on the SAPS or 'flat affect, alogia, avolition and apathy; and anhedonia' on the SANS respectively. Remission status was based on the symptom severity criterion every month. The total duration of positive, negative and total remission during the 2year period was calculated for each participant on the basis of repeated symptom ratings at baseline and months $2,3,6,12,18$ and 24 . The cumulative duration of symptom remission has been reported to be a strong predictor of functional (occupational and social) outcomes. ${ }^{22}$ For missing data, ratings at each time point were carried forward for the next period for up to a maximum of 6 months. Clinical outcome was assessed using three variables: change in symptom severity over 24 months, duration of remission and remission status at month 24 . Early remission was defined as meeting remission criteria by month 3 . 


\section{Family support}

Family support and the quality of family relationships were based on ratings on the following two questions, derived from the Wisconsin Quality of Life Index-Provider Version ${ }^{23}$ : 'During the past four weeks, this person has received ___ support', assessed on a Likert-type scale, with 1 being infrequent, 2 moderate and 3 good; and 'How would you describe the quality of this person's relationship with his/her family in the last 4 weeks?', assessed on a Likert-type scale from 0 'none' to 5 'excellent'. This was evaluated at months 3,12 and 24. This measure was chosen for its simplicity and its limited potential for cultural bias. This assessment was made about the participant by the service provider (typically the case manager) on the basis of observation and enquiry. The score on the support received was multiplied by the score on the quality of relationship to obtain a weighted score (range 0-15) as a measure of overall family support. Higher scores indicate higher levels of family support.

\section{Training and interrater reliability across sites}

Staff at both sites were rigorously trained during the pilot phase and the current study. Interrater reliability (IRR) was established using four videotaped interviews of participants (two from each site), which were rated on the SAPS and SANS by all raters. The Cronbach's alpha for global subscale scores on the SAPS ranged from 0.93 to 0.99 , and from 0.86 to 0.97 for the SANS. This compares with pilot phase IRRs of $0.82-0.87$ on the PANSS. ${ }^{11}$

\section{Data analyses}

Data analyses were carried out using SPSS 22.0 for Windows. ${ }^{24}$ The two samples were compared using two inferential statistics: independent samples $t$-tests for continuous variables and $\chi^{2}$ for categorical variables. Repeated-measures analysis of variance (R-MANOVA) was used to examine the effect of time and site on symptom severity, controlling for baseline severity of each symptom domain. Any emerging site differences were adjusted for the influence of family support at 3 months. Sensitivity analyses were repeated to account for missing data on family support.

Linear and logistic regressions were conducted to determine predictors of duration and status of remission respectively. To address the hypotheses of the present study, the variables were selected on the basis of differences between the sites: gender (male/female); presence of a comorbid substance use or dependence disorder (yes/no); diagnosis (schizophrenia spectrum disorder/ affective psychosis); age at onset of psychosis; baseline SAPS/ SANS scores; 'early remission status' (yes/no); level of family support; and site (Chennai versus Montreal). We also controlled for baseline remission status. Effect sizes were computed using Cohen's $d$ and partial eta squared $\left(\mathrm{np}^{2}\right)$ and interpreted according to the following guidelines: small: Cohen's $d=0.2$ and $\eta p^{2}=0.01$; medium: Cohen's $d=0.5$ and $\eta \mathrm{p}^{2}=0.06$; large: Cohen's $d=0.8$ and $\eta \mathrm{p}^{2}=0.14 .^{25,26}$ All multivariate analyses were carried out on participants who completed the study (supplementary Fig. 1).

\section{Results}

\section{Baseline sociodemographic and clinical characteristics}

Participants at both sites were in their mid-twenties, although significantly younger and more often male and single in Montreal than in Chennai (Table 1). Most participants in Montreal and almost all in Chennai lived with their families. For occupational status, a substantial proportion of the women in the Chennai sample described their full-time role as homemakers.
Most participants had a primary diagnosis of schizophreniaspectrum disorder, albeit significantly more in Chennai. A significantly higher proportion had a concurrent substance use disorder (mostly cannabis) in Montreal. Age at onset was significantly lower in Montreal, although there was no difference in DUP between the two sites. At baseline, participants in Montreal showed a significantly higher level of positive symptoms but no differences were observed on severity of negative symptoms.

A comparison of individuals included in the study and those who declined to participate $(n=35)$ in Montreal revealed no significant differences on demographic and clinical characteristics, except for a lower median DUP among non-participants (4 weeks) compared with participants (11.57 weeks). In Chennai, only 6 individuals declined participation. In Montreal 31 (18.8\%) participants did not complete the study, compared with $2(1.2 \%)$ in Chennai. A comparison of completers with non-completers in Montreal did not reveal any differences on any demographic or clinical characteristics except that the proportion with family contact was higher among the completers than non-completers $\left(50\right.$ v. $29.3 \%, \chi^{2}=$ 4.10, d.f. $=1, P=0.043$ )

\section{Medication}

On entry to the programme, almost all participants in Chennai (166/ 168 ) and most participants in Montreal (141/165) were prescribed and agreed to take antipsychotic medication. The daily dose of antipsychotic (chlorpromazine (CPZ) equivalents) $)^{27,28}$ at the start of treatment was higher in Montreal (mean $202.59 \mathrm{mg}$, s.d. $=146.44$ ) compared with Chennai (mean $168.11 \mathrm{mg}$, s.d. $=78.04)(t=2.671$, d.f. $=186.401, P<0.01)$. There were no significant differences in the use of other psychotropic medications. Olanzapine and aripiprazole were the most common antipsychotic medication used in Chennai $(n=111 ; 63.4 \%)$ and Montreal $(n=65,38 \%)$ respectively.

\section{Outcomes}

Mortality

Four participants (2.38\%), all women (age 27-34), died at the Chennai site, all within 3 months of entry to the programme, compared with none in Montreal over the entire 2-year period. One died from recurrence of remitted thyroid cancer and the other three from suicide.

\section{Family support}

Family support (supplementary Table $\mathrm{S} 1$ ) was higher in Chennai than in Montreal at month 3 (mean 9.39, s.d. $=4.25 v$. mean 8.31, s.d. $=$ 3.91), month 12 (mean 10.64 , s.d. $=3.72 v$. mean 8.49 , s.d. $=4.07)$ and month 24 (mean 10.6, s.d. $=3.41 v$. mean 8.79, s.d. $=3.46$ ). There was an effect of time $\left(F=7.007\right.$, d.f. $=1.915, P<0.001, \eta \mathrm{p}^{2}=$ $0.03)$ and site $\left(F=14.05\right.$, d.f. $\left.=1, P<0.001, \eta p^{2}=0.06\right)$ but no time $\times$ site interaction $\left(F=2.56\right.$, d.f. $\left.=1.915, P=0.08, \eta p^{2}=0.01\right)$. Family support increased over time at both sites.

\section{Decrease in positive and negative symptom severity}

Results of R-MANOVA showed that for both positive and negative symptoms, there were significant main and interaction effects of time and site (Table 2). Participants at both sites improved significantly over the 24 months. However, the improvement was greater in Montreal for positive symptoms $\left(\eta p^{2}=0.19\right)$ and in Chennai for negative symptoms $\left(n \mathrm{p}^{2}=0.08\right)$. This analysis controlled for baseline symptoms. The time $\times$ site interactions reported in Table 2 were adjusted for the effect of family support at month 3, the latter being the main variable of interest that might account for site differences. Following this adjustment, the interaction remained significant for negative symptoms $(F=3.95$ (d.f. $=1.49), P<0.03$, 


\begin{tabular}{|c|c|c|c|c|}
\hline Variable & Montreal & Chennai & Statistical test & $P$ \\
\hline & $<0.001$ \\
\hline Men & $110(66.7)$ & $82(48.8)$ & \multirow[t]{4}{*}{$\chi^{2}(2)=12.37$} & \multirow[t]{4}{*}{0.002} \\
\hline Women & $54(32.7)$ & $86(51.2)$ & & \\
\hline Transgender & $1(0.6)$ & 0 & & \\
\hline Total & 165 & 168 & & \\
\hline Education, years: mean (s.d.) & $12.24(2.63)$ & $11.75(3.9)$ & $t(293.938)=1.34$ & 0.182 \\
\hline \multicolumn{5}{|l|}{ Education level, $n(\%)$} \\
\hline Less than high school & $44(27.2)$ & $47(28)$ & \multirow[t]{3}{*}{$\chi^{2}(1)=0.03$} & \multirow[t]{3}{*}{0.868} \\
\hline High school or more & $118(72.8)$ & $121(72)$ & & \\
\hline Total & 162 & 168 & & \\
\hline \multicolumn{5}{|l|}{ Occupational status, $n$ (\%) } \\
\hline Student & $40(29.0)$ & $24(14.4)$ & \multirow[t]{5}{*}{$\chi^{2}(3)=30.0$} & \multirow[t]{5}{*}{$<0.001$} \\
\hline Paid employment & $35(25.3)$ & $25(15.0)$ & & \\
\hline Homemaker & $7(5.1)$ & $40(24.0)$ & & \\
\hline Unemployed & $56(40.6)$ & $78(46.7)$ & & \\
\hline Total & 138 & 167 & & \\
\hline \multicolumn{5}{|l|}{ Marital status, $n(\%)$} \\
\hline Single & 149 (90.9) & 95 (56.5) & \multirow[t]{4}{*}{$\chi^{2}(2)=50.51$} & \multirow[t]{4}{*}{$<0.001$} \\
\hline Married or common law relationship & $13(7.9)$ & $62(36.9)$ & & \\
\hline Separated, divorced or widowed & $2(1.2)$ & $11(6.5)$ & & \\
\hline Total & 164 & 168 & & \\
\hline \multicolumn{5}{|l|}{ Living situation, $n(\%)$} \\
\hline Alone & $16(10.0)$ & $2(1.4)$ & \multirow[t]{5}{*}{$\chi^{2}(3)=22.95$} & \multirow[t]{5}{*}{$<0.001$} \\
\hline With family & $125(78.1)$ & $140(96.6)$ & & \\
\hline With friend or roommate & $16(10.0)$ & $2(1.4)$ & & \\
\hline In residence, group home or homeless & $3(1.9)$ & $1(0.7)$ & & \\
\hline Total & 160 & 145 & & \\
\hline \multicolumn{5}{|l|}{ SCID diagnosis type, $n$ (\%) } \\
\hline Schizophrenia-spectrum disorder & 109 (67.3) & $150(90.4)$ & \multirow{3}{*}{$\chi^{2}(1)=26.29$} & \multirow[t]{3}{*}{$<0.001$} \\
\hline Affective psychosis & $53(32.7)$ & $16(9.6)$ & & \\
\hline Total & 162 & 166 & & \\
\hline \multicolumn{5}{|l|}{ Substance misuse or dependence (SCID), $n$ (\%) } \\
\hline Yes & $54(37.8)$ & $17(10.2)$ & \multirow[t]{3}{*}{$\chi^{2}(1)=32.9$} & \multirow[t]{3}{*}{$<0.001$} \\
\hline No & $89(62.2)$ & $149(89.8)$ & & \\
\hline Total & 143 & 166 & & \\
\hline Age at onset of current psychotic episode, years: mean (s.d.) & $23.41(5.67)$ & $25.81(5.22)$ & $t(318)=3.94$ & $<0.001$ \\
\hline \multirow[t]{2}{*}{ DUP to presenting episode, weeks: ${ }^{\text {b }}$ mean (s.d.) median (range) } & $40.79(88.46)$ & $32.82(61.09)$ & \multirow[t]{2}{*}{$t(270.4)=0.42^{b}$} & \multirow[t]{2}{*}{0.674} \\
\hline & $9.9(0-684.3)$ & $11.8(0.29-518.71)$ & & \\
\hline SAPS score, mean (s.d.) & $34.53(14.91)$ & $19.90(9.92$ & $t(259.558)=10.192$ & $<0.001$ \\
\hline SANS score, mean (s.d.) & $22.5(12.47)$ & $21.62(15.72)$ & $t(259.18)=0.549$ & 0.583 \\
\hline
\end{tabular}

$\left.\eta \mathrm{p}^{2}=0.20\right)$ and for positive symptoms $(F=54.33$ (d.f. $=1.51), P<$ $\left.0.001, \eta^{2}=0.21\right)$. These results suggest that the greater reduction of negative and positive symptoms in Chennai and Montreal respectively is independent of family support. In the case of negative symptoms, it may be enhanced by such support.

\section{Duration of remission}

Over the 24-month period, the average duration of positive symptom remission was similar in Montreal (mean 15.53 months, s.d. =7.46) and Chennai (mean 15.65 months, s.d. =7.93) $(t=$ -0.13 , d.f. $=331, P=0.89$, Cohen's $d=-0.01$ ). On the other hand, compared with Montreal, participants in Chennai were in negative symptom remission for a significantly longer period (mean 16.10 months, s.d. $=7.56 v$. mean 9.78 months, s.d. $=8.13)(t=-7.35$, d.f. $=331, P<0.001$, Cohen's $d=-0.80$ ).

Is the greater duration of negative symptom remission in Chennai independent of other confounders?

Multiple linear regression analysis was conducted only to establish whether any of the differences between the two samples (Table 1) contributed to the difference in duration of negative symptoms, independent of site. The results of the linear regression $(n=236)$ indicated that attaining early negative symptom remission and site (Chennai) significantly predicted a longer duration of negative symptom remission and accounted for $45 \%$ of variance in outcome (Table 3).

To further explore the relationship between family support and duration of negative symptom remission, bivariate correlations with family support at each site revealed modest correlation at months 3 and 12 in Chennai $(r=0.31)$ and only at month 12 in Montreal $(r=0.26)$.

\section{Remission status at 24 months}

The percentage of participants in positive and negative symptom remission increased over the 2 -year period at both sites. The percentage of participants in positive symptom remission at month 24 was comparable between Montreal and Chennai (82.6 and 83.3\% respectively; $\chi^{2}=0.33$, d.f. $=1, P=0.86$ ), whereas only $60.3 \%$ of participants in Montreal were in negative symptom remission compared with $81.5 \%$ in Chennai $\left(\chi^{2}=16.12\right.$, d.f. $\left.=1, P<0.001\right)$. 
Are site differences in negative symptom remission status at month 24 explained by differences in other characteristics between the two samples?

As there was no difference in rate of positive symptom remission between the two sites, binary logistic regression analyses were used to examine this question for negative symptom remission status only. This analysis $(n=216)$ revealed that only early remission (higher rate in Chennai) of negative symptoms was an independent predictor of remission status at 24 months. Neither site nor family support accounted for this difference (Table 3 ).

\section{Early remission and impact on differences in outcome}

'Early remission' (by month 3 ) appears to be strongly and consistently associated with better outcome for duration of negative symptom remission as well as remission status at 24 months, independent of site. In Chennai 141 (84\%) participants were in negative symptom remission at 3 months compared with 65 (39\%) in Montreal $\left(\chi^{2}=69.98, P<0.001\right)$. Therefore, we conducted a logistic regression with early negative symptom remission as the dependent variable and characteristics generally associated with better outcome on negative symptoms as independent variables (Table 4). Our results show that early negative symptom remission was independently associated with shorter DUP and site (Chennai) and only marginally by not having comorbid substance misuse. Given that there was no significant difference in DUP across the two sites, it can be concluded that the difference in the rate of early remission of negative symptoms is associated with site alone.

\section{Discussion}

We had hypothesised that clinical outcomes would be better in Chennai than in Montreal. This was confirmed for negative but not for positive symptoms. Positive symptoms respond well to antipsychotic medication, especially in an EIS environment, as indicated by previous reports of high rates of remission (80-85\%). ${ }^{15}$ This appears to be the case in both settings. Indeed, the higher magnitude of improvement in positive symptoms in Montreal could very likely be the result of regression to the mean.

Better negative symptom outcomes reported in EIS compared with regular care are likely related to the integration of several psychosocial interventions with medication. ${ }^{29}$ Our results show that in addition to site (Chennai), the better outcomes on negative symptoms are associated with greater family support at 3 months in the case of reduction in symptoms over time and by higher rates of early negative symptom remission (within 3 months) in the case of duration of remission. On the other hand, the status of being in remission at 24 months is associated mostly with the higher rate of early remission in Chennai. Achieving early remission of negative symptoms seems to be a key ingredient for better outcome on negative symptoms. Our examination of the predictors of early remission suggests that this is driven largely by site and DUP, the latter being the same across the two sites. These results further confirm our first hypothesis of better outcome in the EIS in Chennai compared with the one in Montreal, but only for negative symptoms.

We also demonstrated that family support early on (at 3 months), which was higher in Chennai, further enhances better negative symptom outcome independent of site. Family support, however, increases with time at both sites and may contribute to better outcomes in Montreal as well, if increased sufficiently and early enough.

Our results suggest that there may indeed be something other than, or in addition to, family support that influences both early 


\begin{tabular}{|c|c|c|c|c|c|c|}
\hline \multirow[b]{2}{*}{ Predictors for linear regression } & \multicolumn{3}{|c|}{$\begin{array}{c}\text { Duration of remission ( } n \\
=236)\end{array}$} & \multirow[b]{2}{*}{ Predictors for logistic regression } & \multicolumn{2}{|c|}{$\begin{array}{c}\text { Remission status at } \\
\text { month } \\
24(n=216)\end{array}$} \\
\hline & $\beta$ & s.e. & $P$ & & OR $(95 \% \mathrm{Cl})$ & $P$ \\
\hline Gender & 0.38 & 0.85 & 0.652 & Gender (ref: female) & $1.14(0.56-2.33)$ & 0.704 \\
\hline Substance misuse/dependence at baseline & 0.27 & 1.04 & 0.795 & Substance misuse/dependence at baseline (ref: no) & $1.45(0.64-3.26)$ & 0.368 \\
\hline Age at onset of psychosis & -0.01 & 0.07 & 0.881 & Age at onset of psychosis & $1.00(0.94-1.06)$ & 0.916 \\
\hline Diagnosis & 1.00 & 1.01 & 0.322 & Diagnosis (ref: affective psychosis) & $1.24(0.55-2.81)$ & 0.602 \\
\hline Baseline negative remission & -2.01 & 1.07 & 0.063 & Negative remission status at baseline (ref: no) & $0.47(0.16-1.37)$ & 0.172 \\
\hline Early negative remission & -9.04 & 1.03 & $<0.001$ & Early negative remission (ref: no) & $0.38(0.17-0.82)$ & 0.014 \\
\hline Family support at month 3 & 0.13 & 0.09 & 0.177 & Family support at month 3 & $0.95(0.87-1.03)$ & 0.248 \\
\hline Site & 2.79 & 1.04 & 0.008 & Site (ref: Chennai) & $1.48(0.66-3.32)$ & 0.335 \\
\hline Constant & 22.74 & 3.96 & $<0.001$ & Constant & 0.63 & 0.683 \\
\hline Adjusted $R^{2}$ & 0.45 & & & & & \\
\hline
\end{tabular}

remission as well as its sustenance in Chennai. Our family support measure may not have captured nuances of family involvement. There may be other unexplored contextual aspects of family involvement or some other patient or service characteristics that explain inter-site differences in negative symptom outcome. Other inter-site differences may pertain to the trust of patients and families in treatment providers and attributions of relative responsibility for care to patients, families and the state. ${ }^{30,31}$

Our model explained a considerable proportion of variance (45\%) in duration of negative symptom remission and suggests that characteristics that differentiate the EIS in Chennai from that in Montreal make up the 'site' variable and could be influencing the outcome. However, there are likely to be other unexplored variables that contribute to this difference. A substantial amount of unexplained variance may be attributable to individual variations across participants related to genetic or other neurobiological or environmental factors not accounted for in this model (e.g. social deprivation, history of trauma). ${ }^{32,33}$

Better outcome for negative symptoms in Chennai is unlikely to be related to differences in baseline symptom levels, as these were controlled for in our analyses. Variation in the rating of negative symptoms across the two sites is also unlikely to explain this difference, given the high level of interrater reliability on measurement of negative symptoms across the two sites. Although a comorbid substance misuse diagnosis did not contribute significantly to any of the regression models, we did not measure continuous use of cannabis over time. Very low rates of drug use in Chennai have also been reported in previous studies. ${ }^{34,35}$

\section{Strengths}

Our study has several strengths. The samples of patients with essentially untreated FEP at both sites were very well characterised, met the same inclusion and exclusion criteria and received treatment

Table 4 Logistic regression analysis to examine predictors of early negative remission

\begin{tabular}{|llr|} 
& \multicolumn{2}{c|}{$\begin{array}{c}\text { Early negative remission } \\
\text { status }\end{array}$} \\
\cline { 2 - 3 } Predictors & \multicolumn{1}{c|}{ OR $(95 \% \mathrm{CI})$} & $P$ \\
Gender & $2.26(1.16-4.38)$ & 0.016 \\
Substance misuse/dependence at baseline & $0.62(0.28-1.37)$ & 0.246 \\
Age at onset of psychosis & $1.00(0.94-1.07)$ & 0.793 \\
Duration of untreated psychosis (analysis on & $2.38(1.41-4.00)$ & 0.001 \\
$\quad$ & & \\
Diagnosis & $0.75(0.33-1.70)$ & 0.495 \\
Early adherence percentage & $0.99(0.98-1.00)$ & 0.534 \\
Site & $8.86(4.16-18.86)$ & $<0.001$ \\
Constant & 0.09 & 0.052 \\
\hline
\end{tabular}

in an EIS with components of treatment that were similar within respective cultural and economic contexts. Considerable time and resources had been used in building capacity at the Chennai site during the pilot phase. Rigorous training of staff and regular evaluation of interrater reliability were conducted across the two sites.

\section{Limitations}

Differences in service structures may have resulted in participants in Montreal being more representative of a catchment area sample compared with those in Chennai. In Chennai, a large metropolitan area in a LMIC, participants and families may have come from a much larger and wider-spread population base. Participant characteristics varied across the two sites and may reflect true differences in the epidemiology of psychotic disorders in India and Canada (e.g. difference in gender distribution, age at onset and substance misuse) or sampling bias (severity of symptoms). However, these confounders did not explain the site differences in our multivariate analyses. Our controlling for all baseline differences between the two samples is likely to have minimised but not eliminated bias, which must be acknowledged in interpreting differences.

\section{Implications}

Our findings suggest that an EIS with essential elements of integrated care, including case management, contextualised to local circumstances of a LMIC urban environment can produce clinical outcomes that are even better than those in an urban setting in an HIC. The differences in outcome in FEP across the two very different economic and cultural environments are not attributable to differences in some known predictors of outcome that may apply across disparate environments. Clinicians may need to pay particular attention to achieving early remission of symptoms by addressing factors associated with it, while also being vigilant about risk of suicide, especially for women in India. Increasing family support and reducing treatment delay may lead to both early remission and improvements in longer-term outcome, across geographies. Improving family support early in the treatment of young people with FEP may be necessary in Western settings such as Montreal, where family involvement may not occur as naturally as in LMIC settings such as Chennai. There may indeed be a dose-response relationship between family support and outcome, and family support may need to be sustained over the entire period of treatment.

Ashok Malla (D) MBBS, MRCPsych, FRCPC, Department of Psychiatry, MCGill University, Montreal; and Prevention and Early Intervention Program for Psychoses (PEPP-Montreal), Douglas Mental Health University Institute, Montreal, Canada; Srividya N. Iyer (1D), PhD, Department of Psychiatry, McGill University, Montreal, Canada;

Thara Rangaswamy (D), MD, PhD, Schizophrenia Research Foundation (SCARF), Chennai, India; Padmavati Ramachandran (D), MD, Schizophrenia Research 
Foundation (SCARF), Chennai, India; Greeshma Mohan (D), MA, Schizophrenia Research Foundation (SCARF), Chennai, India; Aarati Taksal (D), PhD, Prevention and Early Intervention Program for Psychoses (PEPP-Montreal), Douglas Mental Health University Institute, Montreal, Canada; Howard C. Margolese (D, MD, CM, MSC, FRCPC Department of Psychiatry, McGill University, Canada; Norbert Schmitz (D), PhD, Department of Psychiatry, McGill University, Canada; Ridha Joober (D, MD, PhD, Department of Psychiatry, McGill University, Canada

Correspondence: Ashok Malla. Email: ashok.malla@mcgill.ca

First received 31 Oct 2019, final revision 1 Jun 2020, accepted 9 Jun 2020

\section{Supplementary material}

Supplementary material is available online at https://doi.org/10.1192/bjp.2020.126.

\section{Data availability}

The data that support the findings of this study are available from the corresponding author, A.M., upon reasonable request.

\section{Acknowledgements}

We thank Megan Pope, Heleen Loohuis, Nicole Pawliuk, Kevin MacDonald, Emily Schorr, Helen Martin and service providers, patients and families at PEPP, SCARF and PEPP-MUHC.

\section{Author contributions}

Study design and protocol: A.M., S.N.I., R.T., R.P., R.J., N.S.; implementation and data acquistion: S.N.I., G.M., A.M., R.P., R.J., H.C.M.; data analysis: A.T.; statistical input and interpretation:

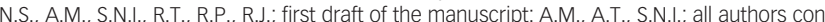
tributed to several drafts and have approved the final manuscript.

\section{Funding}

This research was funded by an R-01 grant from the National Institutes of Health (NIH 5R01MH093303-05). The funding source had no further role in study design, data collection and analysis, writing of the report, and in the decision to submit the report for publication.

\section{Declaration of interest}

A.M. served as a research consultant to and gave lectures at conferences supported by Lundbeck and Otsuka and was on an advisory board meeting for those two companies, for which he received honoraria. S.I. reports grant and salary awards from the Canadian Institutes for Health Research and Fonds de recherche du Québec-Santé, outside the submitted work. H.C.M. reports grants from Acadia, Amgen and SyneuRx international; grants, honoraria and. H.C.M. recials non-financial support from Pfizer; honoraria from Sunovion, Purdue, HLS therapeutics, Mylan
and Shire within the past 3 years, unrelated to the submitted work. R.J. served as speaker and member of advisory board committees for Pfizer, Janssen, BMS, Sunovian, Myelin, Otsuka, Lundbeck, Shire and Perdue; he also received grants from Janssen, BMS, Otsuka, Lundbeck, Astra Zeneca and HLS, within the past 3 years, unrelated to the submitted work.

ICMJE forms are in the supplementary material, available online at https://doi.org/10.1192/ bjp.2020.126.

\section{References}

1 Correll CU, Galling B, Pawar A, Krivko A, Bonetto C, Ruggeri M, et al. comparison of early intervention services vs treatment as usual for early-phase psychosis: a systematic review, meta-analysis, and meta-regression. JAMA Psychiatry 2018; 75: 555-65

2 Malla A, Norman R, McLean T, Scholten D, Townsend L. A Canadian programme for early intervention in non-affective psychotic disorders. Aust N Z J Psychiatry 2003; 37: 407-13.

3 Harrison G, Hopper K, Craig T, Laska E, Siegel C, Wanderling J, et al. Recovery from psychotic illness: a 15-and 25-year international follow-up study. $\mathrm{Br}$ Psychiatry 2001; 178: 506-17.

4 Hopper K, Wanderling J. Revisiting the developed versus developing country distinction in course and outcome in schizophrenia: results from ISOS, the WHO collaborative followup project. Schizophr Bull 2000; 26: 835-46.

5 Lin K-M, Kleinman AM. Psychopathology and clinical course of schizophrenia: a cross-cultural perspective. Schizophr Bull 1988; 14: 555-67.

6 Jablensky A, Sartorius N, Ernberg G, Anker M, Korten A, Cooper JE, et al. Schizophrenia: manifestations, incidence and course in different cultures: a World Health Organization ten-country study. Psychol Med Monogr Supp 1992: 20: 1-97
7 Cohen A, Patel V, Thara R, Gureje O. Questioning an axiom: better prognosis for schizophrenia in the developing world? Schizophr Bull 2008; 34: 229-44.

8 Kulhara P, Chakrabarti S. Culture and schizophrenia and other psychotic disorders. Psychiatr Clin North Am 2001; 24: 449-64.

9 Gayer-Anderson C, Morgan C. Social networks, support and early psychosis: a systematic review. Epidemiol Psychiatr Sci 2013; 22: 131-46.

10 Nunley $M$. The involvement of families in Indian psychiatry. Cult Med Psychiatry 1998; 22: 317-53.

11 Iyer SN, Mangala R, Thara R, Malla AK. Preliminary findings from a study of firstepisode psychosis in Montreal, Canada and Chennai, India: comparison of outcomes. Schizophr Res 2010; 121: 227-33.

12 Iyer S, Jordan G, MacDonald K, Joober R, Malla A. Early intervention for psychosis: a Canadian perspective. J Nerv Ment Dis 2015; 203: 356-64.

13 American Psychiatric Association. Diagnostic and Statistical Manual of Mental Disorders (4th ed text revision) (DSM-IV-TR). APA, 2000.

14 First MB, Spitzer RL, Gibbon M, Williams JB. Structured Clinical Interview for DSM-IV-TR Axis I Disorders: Research Version, Patient Edition (SCID-I/P). Biometrics Research, New York State Psychiatric Institute, 2002.

15 Malla A, Norman R, Schmitz N, Manchanda R, BÉChard-Evans L, Takhar J, et al. Predictors of rate and time to remission in first-episode psychosis: a two-year outcome study. Psychol Med 2006; 36: 649-58.

16 Andreasen NC. Scale for the Assessment of Positive Symptoms (SAPS). University of lowa, 1984.

17 Andreasen N. The scale for the Assessment of Negative Symptoms (SANS). University of lowa, 1983.

18 Malla AK, Norman RMG, Williamson $\mathrm{P}$, Cortese L, Diaz F. Three syndrome concept of schizophrenia: a factor analytic study. Schizophr Res 1993; 10: 143-50.

19 Cassidy CM, Rabinovitch M, Schmitz N, Joober R, Malla A. A comparison study of multiple measures of adherence to antipsychotic medication in first-episode psychosis. J Clin Psychopharmacol 2010; 30: 64-7.

20 Jordan G, Lutgens D, Joober R, Lepage M, lyer SN, Malla A. The relative contribution of cognition and symptomatic remission to functional outcome following treatment of a first episode of psychosis. J Clin Psychiatry 2014; 75 e566-72.

21 Andreasen NC, Carpenter Jr WT, Kane JM, Lasser RA, Marder SR, Weinberger DR. Remission in schizophrenia: proposed criteria and rationale for consensus. Am J Psychiatry 2005; 162: 441-9.

22 Jordan G, Veru F, Lepage M, Joober R, Malla A, Iyer SN. Pathways to functional outcomes following a first episode of psychosis: The roles of premorbid adjustment, verbal memory and symptom remission. Aust $\mathrm{N} Z \mathrm{~J}$ Psychiatry 2018; 52: 793-803.

23 Becker MA, Shaw BR, Reib LM. Quality of Life Assessment Manual. University of Wisconsin, 1995.

24 IBM C. IBM SPSS Statistics for Windows 22.0. IBM Corp, 2013

25 Cohen J. Statistical Power Analysis for the Behavioral Sciences (2nd edn). Lawrence Erlbaum, 1988.

26 Gray CD, Kinnear PR. IBM SPSS Statistics 19 Made Simple. Psychology Press, 2012

27 Woods SW. Chlorpromazine equivalent doses for the newer atypical antipsychotics. J Clin Psychiatry 2003; 64: 663-7.

28 Leucht S, Samara M, Heres S, Davis JM. Dose equivalents for antipsychotic drugs: the DDD method. Schizophr Bull 2016; 42(suppl 1): S90-4.

29 Lutgens D, Gariepy G, Malla A. Psychological and psychosocial interventions for negative symptoms in psychosis: systematic review and meta-analysis. $\mathrm{Br} J$ Psychiatry 2017; 210: 324-32.

30 Pope MA, Jordan G, Venkataraman S, Malla AK, Iyer SN "Everyone has a role": perspectives of service users with first-episode psychosis, family caregivers, treatment providers, and policymakers on responsibility for supporting individuals with mental health problems. Qual Health Res 2019; 29: 1299-312.

31 Pope MA, Malla AK, Iyer SN. Who should be responsible for supporting individuals with mental health problems? A critical literature review. Int J Soc Psychiatry 2018; 64: 293-302.

32 Suvisaari J, Mantere $\mathrm{O}$, Keinänen J, Mäntylä T, Rikandi E, Lindgren $\mathrm{M}$, et al. Is it possible to predict the future in first-episode psychosis? Front Psychiatry 2018; 9: 580.

33 Díaz-Caneja CM, Pina-Camacho L, Rodríguez-Quiroga A, Fraguas D, Parellada $\mathrm{M}$, Arango $\mathrm{C}$. Predictors of outcome in early-onset psychosis: a systematic review. NPJ Schizophrenia 2015; 1(1): 14005.

34 Thara R. Twenty-year course of schizophrenia: the Madras Longitudinal Study. Can J Psychiatry 2004; 49: 564-9.

35 Chand $\mathrm{P}$, Thirthalli J, Murthy P. Substance use disorders among treatment naïve first-episode psychosis patients. Compr Psychiatry 2014; 55: 165-9. 